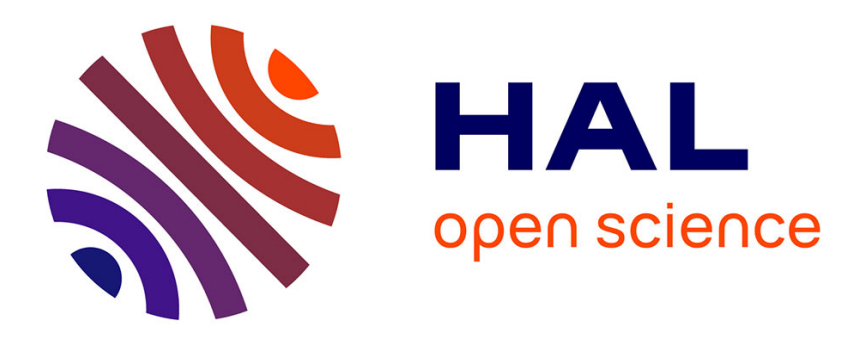

\title{
Poincaré et la déconstruction du négatif Thierry Paul
}

\section{- To cite this version:}

Thierry Paul. Poincaré et la déconstruction du négatif. Les mutations de l'écriture, Presses de la

Sorbonne, 2013. hal-00624900

\section{HAL Id: hal-00624900 \\ https://hal.science/hal-00624900}

Submitted on 20 Sep 2011

HAL is a multi-disciplinary open access archive for the deposit and dissemination of scientific research documents, whether they are published or not. The documents may come from teaching and research institutions in France or abroad, or from public or private research centers.
L'archive ouverte pluridisciplinaire HAL, est destinée au dépôt et à la diffusion de documents scientifiques de niveau recherche, publiés ou non, émanant des établissements d'enseignement et de recherche français ou étrangers, des laboratoires publics ou privés. 


\title{
POINCARÉ ET LA DÉCONSTRUCTION DU NÉGATIF
}

\author{
THIERRY PAUL
}

TABLE DES MATIÈRES

1. Introduction 2

2. Les problèmes qui troublent Poincaré 2

2.1. La Mécanique Céleste 3

2.2. La Mécanique Quantique 3

3. Les solutions négatives 4

3.1. De la non-convergence à la divergence 4

3.2. Une preuve de la nécessité de l'hypothèse de Planck 5

4. L'écriture 7

4.1. L'écriture de la divergence dans le paradigme analytique 7

4.2. L'écriture du discret dans le continu; la masse de Dirac 8

4.3. KAM et Heisenberg : deux nouvelles écritures 8

5. Une méthodologie de la déconstruction $\quad 8$

5.1. Déconstruction d'une série 8

5.2. Déconstruction du raisonnement dans le continu 9

5.3. KAM et le discret, Heisenberg et la mécanique céleste, deux
reconstructions

6. De la déconstruction du négatif à la construction de nouveaux
paradigmes

6.1. La perte du paradigme Laplacien : de la divergence au chaos $\quad 9$

6.2. (vers) La perte du paradigme de l'espace absolu : du discret au $\begin{array}{ll}\text { quantique } & 10\end{array}$

7. Lien avec la déconstruction de Derrida? 10

8. Conclusion : la quête de l'écriture du "non" comme moteur vers de nouveaux paradigmes 11

Annexe A. Best...iaire/-off du négatif chez Poincaré 11

$\begin{array}{ll}\text { Annexe B. Le calcul comme écriture } & 12\end{array}$

Annexe C. Écriture et changement de paradigme en musique? 12

$\begin{array}{ll}\text { Références } & 12\end{array}$ 


\section{INTRODUCTION}

"J'attire l'attention du lecteur sur les résultats négatifs présents dans ce mémoire...". C'est par ces mots que Poincaré termine l'introduction de son mémoire sur les trois corps, second mémoire succédant à un premier, positif celui-là, et incorrect.

Les résultats négatifs traversent la vie de Poincaré jusqu'à ses ultimes articles, jusqu'à celui sur les quanta dont l'introduction se termine par :"je suis arrivé à un résultat négatif...".

Poincaré conservateur, mais obsédé par les "raisons" du négatif, s'intéresse à celui-ci pour se convaincre lui-même de l'échec des raisonnements qui n'aboutissent pas.

"Divergences des séries" écrit-il dans la table des matières du deuxième tome des "Méthodes nouvelles", qui contient ce qu'il appellera une description des résultats des astronomes, et qui est en fait une réécriture, une écriture même, peut-être, de l'astronomie moderne.

Car il s'agit bien ici d'écriture : au sens littéral tout d'abord, écriture des séries (phrases écrites dans l'alphabet des signes mathématiques, et qui en sont rien d'autre que l'algorithme qu'elles représentent (mais un nombre est-il autre-chose qu'une écriture?)) et écriture du discret dans le continu comme nous le verrons pour les quanta. Mais aussi écriture dans la transmission de la science, si positivement considérée pourtant. En effet l'écriture "en négatif" du résultat, si fréquente, par exemple, en musique ("pas trop fort", "moins"...) fait référence à une dynamique, tout comme le font les résultats négatifs de Poincaré, dans une dynamique du paradigme changeant, de la théorie qui évolue. Les résultats négatifs de la Mécanique Céleste se transforment en étude positive du chaos, ceux de l'article sur les quanta en nécessité de l'hypothèse de Planck.

Mais pour se convaincre que le négatif de l'un fait le positif de l'autre, il faut une compréhension de la raison du négatif, de sa raison d'être absolue, de son incontournabilité qui ne laisse, finalement, entrevoir que le changement de paradigme. Il faut se convaincre de l'absolue nécessité du négatif tout d'abord, et ensuite, par l'analyse de celui-ci, pouvoir pousser plus loin pour "positiver" ; bref, il faut une méthode.

Poincaré l'a utilisée au moins deux fois; en Mécanique Céleste pour comprendre les divergences, en Mécanique Quantique pour comprendre le discret. Dans les deux cas il a réduit les calculs, les a séparés, bref il a analysé pierre par pierre les édifices existants pour voir les points de friction.

Cette méthode nous semble, plus d'un siècle après, avoir un nom : la déconstruction.

\section{Les Problèmes qui troublent Poincaré}

Aux deux extrémités de sa carrière, Poincaré va s'intéresser à deux problèmes emblématiques de la physique : la mécanique céleste et la théorie quantique, ce dernier intérêt brutalement interrompu par sa mort. 
2.1. La Mécanique Céleste. Le fameux problème des trois corps place tout de suite l'activité de Poincaré dans une dynamique qui va effectuer une véritable révolution scientifique, et plus tard dans le monde des idées. Il s'agit de savoir si le système planétaire comportant seulement trois planètes est "intégrable" ou pas. Le système à deux planètes l'est, se réduisant au cas monocorpusculaire grâce à l'usage du repère du centre de masse. Le problème des trois corps "réduit", planaire, était à l'époque où Poincaré s'y intéresse, un défi scientifique important : en plus du défi technique, c'était bien le paradigme de stabilité laplacienne qui était en jeu. Il s'agissait de savoir si une petite perturbation d'un système stable aller entamer cette stabilité si chère à Laplace.

Les astronomes avaient depuis longtemps abordé ce problème de façon perturbative, pas des développement en série, témoignage d'un paradigme de l'analytique. La convergence de telles séries non seulement n'était même pas évoquée par eux, mais la difficulté résidait déjà dans l'obtention explicite de telles séries, des termes de ces séries. Poincaré pensa tout d'abord avoir montré leur convergence, et obtint le fameux prix du roi de Suède, puis on s'aperçut d'une erreur, et nous verrons plus loin l'attitude qu'adopta le grand mathématicien. Mais la première prise en charge du problème par Poincaré fut de comprendre le sens même d'une série perturbative.

Ainsi pour prendre un exemple simple, considérons les deux séries qui ont pour terme général :

$$
\frac{1000^{n}}{1.2 .3 \ldots n} \text { et } \frac{1.2 .3 \ldots n}{1000^{n}}
$$

Les géomètres ${ }^{1}$ diront que la première série converge, et même qu'elle converge rapidement, parce que le millionième terme est beaucoup plus petit que le 999999e; mais regarderons la seconde comme divergente, parce que le terme général peut crô̂tre au delà de toute limite.

Les astronomes ${ }^{2}$, au contraire, regarderons la première série comme divergente, parce que les 1000 premiers termes vont en croissant, et la seconde comme convergente, parce que les 1000 premiers termes vont en décroissant et que cette décroissance est d'abord très rapide.

Poincaré s'intéressera très tôt à ces séries dans le cadre de l'astronomie et tentera de montrer leurs convergences, étant lui-même conservateur et personne à l'époque n’imaginant de réel problème de ce coté là.

2.2. La Mécanique Quantique. La Théorie Quantique est née en 1900, dans l'article fondateur de Planck. Elle mettra 25 ans à acquérir une forme dynamique.

Poincaré s'intéresse très tard à la théorie des quanta. Invité au Congrès Solvay de 1911, il découvrit la problématique quantique et écrivit alors coup sur coup plusieurs articles sur le sujet. Certains de "vulgarisation" [12], et deux articles scientifiques $[13,14]$ dans lesquels il va s'efforcer de montrer que l'hypothèse de

1. mathématiciens

2. physiciens 
Planck ne peut être contournée et remplacée par une théorie "continue". Il s'agt pour lui de se demander si la formule de Planck donnant la densité d'énergie du corps noir, et dérivée depuis l'hypothèse des quanta (qui consiste, pour dire vite, à remplacer dans un calcul une intégrale par une somme) peut être (ou non) déduite d'un modèle continu d'interaction entre rayonnement et molécules. Le résultat que Poincaré lui-même présentera comme négatif validera la théorie de Planck.

Ces deux articles sont peu cités de nos jours, mais ont eu un retentissement important à l'époque. En est la preuve le fait que deux articles dans le Volume 38 d'Acta Mathematica en hommage à Poincaré y sont consacrés. De plus l'argument de Poincaré sera repris et d'une certaine manière rendu plus rigoureux dans le célèbre traité de Mécanique Statistique de Fowler en 1929 [3]. Leur (re)lecture après plus d'un siècle de Mécanique Quantique est intéressante à plusieurs égards. En particulier ils montrent, à travers une discussion parfaitement technique, l'affrontement entre discret et continu, en ce début de XXième siècle où la problématique fondationnelle des mathématiques fait rage.

\section{LES SOLUTIONS NÉGATIVES}

3.1. De la non-convergence à la divergence. Lorsque Poincaré travaille au problèmes des trois corps, il cherche des solutions données par des intégrales premières analytiques. Sans vouloir rentrer dans les détails, disons que, dans un cadre très restreint, Poincaré cherche à montrer, en accord d'ailleurs avec la plupart de ses contemporains, que le système à trois corps, avec l'une des masses petite, n'est qu'une perturbation inoffensive du système à deux corps qui, lui, est intégrable. Cette dernière assertion veut dire que, dans le cas des deux corps, l'évolution à tout temps est connue explicitement, sans aucun mystère. C'est le grand succès de la mécanique Newtonienne. Et Poincaré cherche des solutions analytiquement proche du système non-perturbé, c'est-à-dire qu'il pense que les calculs perturbatifs vont donner la solution, par une simple convergence des séries invoquées. C'est un résultat très fort. Et c'est la résolution du paradoxe de Zénon : une somme infinie de termes devenant de plus en plus petit peut converger, être elle-même finie.

Dans la citation mentionnée dans le paragraphe précédent, Poincaré a compris qu'une série peut-être utile, et nous verrons plus bas comment, même si elle ne converge pas. C'est la revanche de Zénon. Mais pour l'heure, sûr de sa conviction toute laplacienne, Poincaré pense que le mouvement des trois corps est analytiquement proche de celui des deux corps. Il croit le montrer dans son célèbre mémoire pour le roi de Suède, mais on lui fait remarquer que le texte comporte une erreur, ou plus exactement, et c'est important, un "trou" dans la preuve : la preuve n'est pas complète.

Le changement paradigmatique que va observer Poincaré est tout à fait hallucinant. Au lieu d'essayer (besogneusement) de combler le trou, d'essayer de sauver ce qui est sauvable dans ses écrits, il va brusquement prendre l'attitude inverse, et commencer à montrer que les séries en question ne convergent pas. 
Nous allons essayer plus bas d'expliquer comment cette tentative passe par une analyse bien particulière de l'écriture de ces séries. Mais disons tout de suite que ce changement de point de vue va avoir une incidence immense sur l'évolution de la science : l'abandon du paradigme laplacien, et la possibilité donnée à la théorie du chaos de se développer. Comment cela?

Et bien justement en s'occupant du négatif. Si les séries divergent, il doit y avoir une raison. Et en étudiant non seulement la divergence, mais les raisons de celle-ci, Poincaré va, en quelque sorte, incarner ces raisons, leur donner une ontologie en leur créant un environnement dans lequel elles pourront avoir un sens, et un sens "positif". C'est ce passage du négatif au positif, négatif dans le vieux paradigme laplacien, positif dans le nouveau, celui de la théorie des systèmes dynamiques, qui constitue un changement drastique, un tournant de la science moderne. Nous allons voir plus loin comment, selon nous, ce passage s'est effectué autour de l'écriture, et d'une certaine méthodologie déconstructive des résultats négatifs

3.2. Une preuve de la nécessité de l'hypothèse de Planck. En 1911 Poincaré est invité au prestigieux congrès Solvay. Il y a prend conscience de l'importance de la théorie quantique et s'interroge sur ses fondements. Il écrit alors coup sur coup plusieurs articles sur le sujet. Certains de "vulgarisation" [12], et deux articles scientifiques $[13,14]$ dans lesquels il va s'efforcer de montrer que l'hypothèse de Planck ne peut être contournée et remplacée par une théorie "continue". Il s'agt pour lui de se demander si la formule de Planck donnant la densité d'énergie du corps noir, et dérivée depuis l'hypothèse des quanta (qui consiste, pour dire vite, à remplacer dans un calcul une intégrale par une somme) peut être (ou non) déduite d'un modèle continu d'interaction entre rayonnement et molécules. Le résultat que Poincaré lui-même présentera comme négatif validera la théorie de Planck.

Ces deux articles sont peu cités de nos jours, mais ont eu un retentissement important à l'époque. En est la preuve le fait que deux articles dans le Volume 38 d'Acta Mathematica en hommage à Poincaré y sont consacrés. De plus l'argument de Poincaré sera repris et d'une certaine manière rendu plus rigoureux dans le célèbre traité de Mécanique Statistique de Fowler en 1929 [?]. Leur (re)lecture après plus d'un siècle de Mécanique Quantique est intéressante à plusieurs égards. Tout d'abord ils montrent, à travers une discussion parfaitement technique, l'affrontement entre discret et continu, en ce début de XXième siècle où la problématique fondationnelle des mathématiques fait rage. Ensuite, et justement autour de cette dualité discret/continu, ils présentent de merveilleuses mathématiques. On y pressent en particulier la masse de Dirac, et le lemme de la phase stationnaire, qui deviendra si important par la suite pour la Mécanique Quantique y est utilisé. Enfin et surtout, ils exhibent clairement une part méthodologique de son héritage scientifique : l'intérêt pour les résultats négatifs, pour les erreurs. Depuis l'erreur dans le système des 3 corps jusqu'à son "échec" dans la tentative de remplacer l'hypothèse des quanta Poincaré n'aura cessé de décortiquer le négatif pour y faire naître le constructif. 
1911 se situe dans une période un peu creuse pour la théorie quantique. L'hypothèse des quanta, énoncée par Planck en 1900 et généralisée par Einstein avec l'effet photoélectrique en 1905, est certes bien acceptée, mais l'application à la théorie atomique (Bohr 1913) et surtout l'établissement d'une véritable dynamique quantique (Heisenberg 1925) se font attendre. L'hypothèse des quanta consiste, dans notre langage moderne, à discrétiser une intégrale (ce que fait notre ordinateur), mais sans faire tendre, a posteriori, le pas de discrétisation vers zéro.

L'idée d'une théorie physique qui puiserait ses sources hors du cadre "naturel" des équations différentielles de la culture classique est difficile à admettre au début du XXième siècle. Poincaré prend à bras le corps la problématique quantique : ne serait-il pas possible de dériver la même loi que Planck sans l'hypothèse discrète? Poincaré y consacrera une note et un article, et donnera une réponse négative. Il mourra en 1912. En 1913 Niels Bohr appliquera avec le succès que l'on sait la théorie au modèle planétaire de l'atome, et en 1926 Erwin Schrödinger définira une équation aux dérivées partielles (donc plongée dans le continu), qui n'aura de solution raisonnable que pour des valeurs discrètes des paramètres d'énergie : le retour à une vision du phénomène quantique plongée dans le continu naîtra donc 15 ans après l'article de Poincaré.

Nous n'allons pas ici rappeler la théorie du corps noir ni présenter en détail la contribution de Planck. Disons seulement que le problème consiste à trouver une formule d'interpolation pour la densité d'énergie $u$ d'un corps "noir", exprimée par rapport à la fréquence du rayonnement $\nu$, telle que

$$
u(\nu) \sim \nu^{2}, \nu \rightarrow 0
$$

et

$$
u(\nu) \sim e^{-c t e \nu}, \nu \rightarrow \infty
$$

Une formule simple est celle imaginée par Planck :

$$
u(\nu)=\frac{8 \pi \nu^{2}}{c^{3}} \frac{h \nu}{e^{h \nu / k T}-1} .
$$

C'est cette loi que Planck dérive en 1900 sous l'hypothèse des quanta. Plus exactement il montre que, si l'on suppose que l'énergie de chaque oscillateur est un multiple entier d'une certaine quantité $\epsilon$, donc de la forme $P \epsilon, P$ entier, un calcul combinatoire donne que l'entropie $S$ de chaque oscillateur est une fonction (explicite) de $\frac{U}{\epsilon}$, où $U$ est l'énergie de chaque oscillateur.

Poincaré va reprendre le calcul en partant d'un densité d'énergie générique $w$ et montrer tout d'abord que si $w$ est ce que l'on appellerait aujourd'hui un "peigne de Dirac" sur les entiers $(\times h)$ il retrouve la loi de Planck. C'est donc en quelque sorte une vision plus mathématique, ou plutôt moins thermodynamique, que celle de Planck.

Mais il ne va pas s'arrêter là. Il va surtout faire le chemin inverse, et "montrer" que la forme de la fonction $u$ détermine $w$ de façon univoque, et donc que seule l'hypothèse des quanta donne le bon résultat. 
C'est ce résultat que Poincaré qualifie de négatif. Plus précisément il va encore une fois s'appréhender un problème et le pousser jusqu'au bout. Puisque la distribution "discrète" marche, il va poser la question : est-ce la seule qui "marche"? En faisant cela il va supposer a priori une distribution d'énergie "continue", il va la traiter comme telle, et s'apercevoir que la seule solution est celle qui est "discontinue". nous allons essayer de présenter un peu en détail ce raisonnement dans la section suivante, et montrer qu'il s'agit bien d'un travail sur l'écriture, mais remarquons dès maintenant qu'ici aussi Poincaré a la même attitude vis-à-vis du négatif qu'il avait pour la mécanique céleste.

Il nous semble que ce qui ressort de cette dernière construction, plutôt ce qui aurait pu ressortir sans la mort de Poincaré l'année suivante, est une façon de voir le discret dans le continu des analystes, en donnant sinon, à l'époque, un sens, mais tout du moins une importance, à ces "fonctions" nulles partout sauf en un point, et d'intégrale, de masse, signifiante, disons égale à un. Dirac utilisera abondamment cette fonction delta, qui deviendra une "distribution" sous les doigts de Laurent Schwartz en 1951. C'est bien un véritable changement de paradigme qui s'opère sous nos yeux déjà en 1911.

\section{L'ÉCRITURE}

Nous allons, dans cette section, tenter de montrer comment les deux changements de paradigme évoqués dans la section précédente sont nés dans des calculs, et par un soin particulier apporté à l'examen de l'écriture de ces derniers.

4.1. L'écriture de la divergence dans le paradigme analytique. Une série est une écriture, l'écriture d'une somme infinie. C'est bien sûr l'écriture d'un algorithme, puisque nul n'a le temps de calculer jusqu'à l'infini. Une série n'a de sens classique que dans le contexte de la convergence : une série est convergente lorsque la suite de ses sommes partielles converge. On écrit

$$
\sum_{n=0}^{n=\infty} c_{n} \epsilon^{n}
$$

comme on pourrait écrire autre-chose.

Mais c'est une écriture séduisante, puisque qu'elle écrit de la même façon une somme finie

$$
\sum_{n=0}^{n=N} c_{n} \epsilon^{n}
$$

et l'on remplace "formellement", pour passer de l'une à l'autre, $N$ par $\infty$.

Et justement l'écriture des séries chez les astronomes est seulement formelle, et n'a plus que bien peu en commun avec l'idée d'une somme finie. C'est à dire que, lorsqu'elle diverge, une série n'a plus rien à voir avec une somme finie. On peut parfois "sommer" une série divergente (sommabilité de Borel, par exemple), mais il faut avoir en général une information supplémentaire sur ce qu'est le résultat de la sommation pour donner un sens à la série "resommée". 
De la même façon qu'une série est une écriture, nous verrons plus bas que la démonstration de la divergence d'une série est aussi une histoire d'écriture. C'est ce qu'a découvert Poincaré.

4.2. L'écriture du discret dans le continu ; la masse de Dirac. Tout comme $\sum_{o}^{\infty}$ représente une écriture, puisque on n'effectue jamais la somme jusqu'à l'infini, le signe $\int_{0}^{\infty}$ représente de même une écriture. Mais, pour ce qui est de $\delta$, ce signe représente en quelque sorte deux infinis : l'infini large et l'infini microscopique.

L'infini microscopique car le support de la fonction, lieu de signifiance de cette dernière, est réduit à un point. Et l'infini large puisque l'intégrale de cette "fonction" jusqu'à n'importe quel nombre, y compris l'infini, est constante et égale à un.

On écrit

$$
\delta(x)
$$

comme on écrit

$$
\sum_{n=0}^{n=N} c_{n} \epsilon^{n},
$$

et l'on donne un sens à $\delta(x)$ par rapport à une fonction "gentille" (par exemple un polynôme) comme on donne un sens à $\sum_{n=0}^{n=\infty} c_{n} \epsilon^{n}$ par rapport à une série convergente (par exemple finie).

4.3. KAM et Heisenberg : deux nouvelles écritures. Ce qui caractérise, à mon sens, la démarche de Poincaré , réside dans sa faculté à "mettre sur la table" les calculs ou méthodes existants afin de comprendre ce qui ne marche pas. Dans le cas de la mécanique céleste, il travaille sur les calculs des astronomes, dans le cas de la mécanique quantique, il effectue ce travail dans sa propre démarche. Plus tard en mécanique quantique (1925), et bien plus tard encore en mécanique céleste (1954), le discret prendra ses lettres de noblesse; la mécanique des matrices de Heisenberg écrira la théorie des quanta dans un formalisme unifié, et la théorie de Kolmogorov placera la convergence des séries des astronomes sur un ensemble de Cantor, autre exemple d'objet défini par une écriture. Suprême alliance de la convergence et d'une forme de discret.

\section{UNE MÉTHOdOLOGIE DE LA DÉCONSTRUCTION}

5.1. Déconstruction d'une série. Pour montrer qu'une série converge le mathématicien a l'habitude de borner la série par la série des valeurs absolues de ses propres termes, en vertu de l'inégalité $|a+b| \leq|a|+|b|$. C'est là un moyen parfois efficace, mais peu économe. En effet on a pu dire que la théorie des perturbations se résume à utiliser que $0 \leq 2$ (prendre $a=1, b=-1$ dans l'exemple précédent). D'un autre coté, si l'on veut, dans la dynamique inverse, montrer qu'une série diverge, la divergence de la suite des valeurs absolues ne suffit évidement pas. 
Poincaré montrera que la présence de petits dénominateurs dans les séries des astronomes crée une "dynamique de la divergence". Cette dynamique négative sera soignée dans la théorie KAM par l'ablation récursive des mauvaises valeurs des paramètres, donnant lieu à la construction d'un ensemble de Cantor.

Montrer qu'une série diverge est difficile, et nécessite de mettre à plat l'algorithme même de la construction de la série. Il faut démonter (et non démontrer) le processus de convergence. Mais montrer la divergence d'une série n'est pas seulement une prouesse technique, c'est aussi une attitude psychologique à avoir. Poincaré l'a eue, après qu'on lui ait montré une erreur dans sa preuve de la convergence des séries astronomiques. Nous reviendrons plus bas sur ce point.

5.2. Déconstruction du raisonnement dans le continu. Dans son article sur la mécanique quantique, Poincaré va en quelque sorte déconstruire le raisonnement dans le continu. Il va chercher à prouver une formule éminemment discrète (Planck) dans le paradigme du continu, et faire surgir le discret du continu, par la nécessité de la masse de Dirac. On voit donc là aussi une attitude à contre-courant, et la difficulté liée à une telle dynamique : pour faire surgir en toute rigueur les distributions des fonctions (régulières) il faudra attendre les années cinquante.

Paradoxalement le discret dans le continu est plus difficile que le continu à partir du discret.

5.3. KAM et le discret, Heisenberg et la mécanique céleste, deux reconstructions. La stabilité strictement classique (KAM) passe par une forme de "discret" de l'ensemble des bonnes conditions initiales : disons pour simplifier que les séries astronomiques se mettent à converger pour des valeurs très particulières, ne remplissant pas des ouverts topologiques, de certains paramètres. De la même façon la notion de discret quantique, la théorie des matrices de Heisenberg et un peu plus tard l'équation de Schrödinger, donne un cadre à la théorie des quanta et ...à la stabilité de la mécanique céleste. Quelle belle symétrie.

\section{De la DÉCOnstruction du NÉGATif À LA CONSTRUCTION DE NOUVEAuX PARADIGMES}

La levée de paradoxes a toujours été, bien sûr, le meilleur moyen dynamique qu'a la science d'avancer. Mais les paradoxes apparaissent en général par confrontation avec l'expérience ou avec la structure logique d'une théorie. Dans les deux cas qui nous intéressent ici, rien de pareil. Dans les deux cas il s'agit d'imaginer (ou dans le deuxième plutôt d'entrevoir) un changement de paradigme par l'examen approfondi de ce que l'on n'arrive pas à montrer, de l'échec. Comprendre l'échec, et le transformer en positif. Voyons cela de plus près.

6.1. La perte du paradigme Laplacien : de la divergence au chaos. Car il fallait bien donner un sens à ces divergences. La perte de l'idée de stabilité par perturbations, paradigme laplacien, ne pouvait rester sans réponse, en tous cas pour Poincaré. Il allait tout d'abord, comme nous l'avons vu plus haut, donner 
un statut théorique, mathématique, géométrique dans son vocabulaire, à l'idée même d'une série divergente par la notion de série asymptotique. Ainsi la théorie des perturbations des astronomes serait asymptotique. Mais immédiatement la question allait se poser :

asymptotique, mais à quoi?

La réponse fût : asymptotique à rien, justement. En tous cas à rien ds comparable à la situation non perturbée. D'où la nécessité d'un changement de paradigme. Si les systèmes perturbés n'étaient pas stables, il devaient être instables. Et de fortes perturbations devaient créer de fortes instabilités (par un paradigme laplacien renversé, en quelque sorte) : l'idée de chaos était née. On pourra demander s'il est bien toujours question ici d'écriture. La réponse me semble être clairement affirmative, car il fallait écrire l'instabilité, il fallait exhiber dans l'écriture des séries des astronomes le phénomène qui les faisait diverger.

Il fallait exhiber l'écriture du non.

6.2. (vers) La perte du paradigme de l'espace absolu : du discret au quantique. La physique classique, jusqu'à 1925, repose sur l'idée d'un espace absolu chère à Newton. Cet espace est par essence continu, puisque lieu des trajectoires de la dynamiques indicées par le temps (continu). On voit là l'incidence de la dynamique, donnée par une équation différentielle, sur la construction de l'espace. De cet espace continu va surgir le discret quantique, par résolution de certaines équations différentielles. Au fond c'est Poincaré qui l'a montré le premier, comme résultat négatif de sa tentative de dériver dans le continu la loi de Planck; cette dernière peut bien se démontrer dans le continu, mais avec (et seulement avec) l'aide d'une écriture "fonctionnelle" du discret, avec cette fonction partout nulle sauf en un point et d'intégrale égale à un. Cette masse de Dirac deviendra plus tard une distribution, socle naturel de cette théorie quantique qui aura dû entre-temps abandonner l'idée d'espace absolu, par l'abandon de l'idée même de trajectoire d'une particule.

\section{Lien AVEC LA DÉCONSTRUCTION DE DERRIDA?}

Il semble que la théorie de la déconstruction ait été peu utilisée en sciences. Il y a certainement une raison naturelle à cela dans le fait que l'on considère que la "construction" scientifique est solide.

Il faut d'ailleurs bien remarquer que la démarche de Poincaré est singulière, et fût mal considérée pendant longtemps. Cet intérêt partagé de Poincaré pour le négatif aussi bien que pour le positif n'est pas seulement une singularité dans le paradigme mathématique, mais dans le monde scientifique en général. Elle peut donner l'impression d' "aller contre", comme le fait de revisiter les classiques en les "déconstruisant" a donné certainement lieu à beaucoup de malentendus.

Mais justement la science a peut-être cela de particulier que de pouvoir signer, au sens de positif/négatif, ses résultats. Il ne s'agit pas ici de placer l'un contre 
l'autre ces deux signes, mais de remarquer la puissance (et d'ailleurs par là même les limites) de la science qui réside dans le fait de décider entre le positif et le négatif.

C'est dans cette perspective que se situe l'entreprise de déconstruction de Poincaré. Poincaré ne déconstruit que le négatif, c'est cela qui l'intéresse. Son obsession à comprendre les raisons de l'échec lui a fait découvrir de nouveaux paradigmes, conséquences positives de la déconstruction du négatif, tout comme

$$
-\times-=+ \text {. }
$$

\section{Conclusion : LA QuÊte De L'ÉCRIture du "NON" COMME MOteur Vers DE NOUVEAUX PARADIGMES}

Nous avons voulu montrer dans ce court essai comment Poincaré, à deux époques extrêmes de son existence, a réussi à inventer (pour la première) et pressentir (pour la seconde) de nouveaux paradigmes par une méthode de déconstruction de résultats négatifs établis avant lui. D'un strict point de vue "logique" il n'y a rien d'étonnant à ce que le négatif de la déconstruction s'allie au négatif $d u$ déconstructible pour donner du positif, mais encore faut-il voir ce qu'il y a de déconstructible dans un résultat négatif. Nous avons essayé de montrer que ce que l'on peut déconstruire dans le négatif est justement l'écriture de celui-ci, et non son "sens" même. La divergence d'une série est un fait négatif, inaltérable, "sharp" dirait-on. Et pauvre. Mais le mécanisme qui fait que la série diverge, est, lui, d'une tout autre valeur. Et puisque une série, et en particulier pleinement ses propriétés de convergence, vit dans une dimension infinie (toute série finie converge trivialement), c'est son écriture, son algorithme qu'il faut interroger si l'on veut comprendre et imaginer une suite. Poincaré a compris que les raisons de la divergence des séries des astronomes étaient les traces d'un nouveau paradigme, le chaos.

Il a compris que l'écriture nous révélait l'indicible.

\section{Annexe A. Best... iaire/-OFf du nÉGatif Chez Poincaré}

"J'attire l'attention du lecteur sur les résultats négatifs présents dans ce mémoire..." "je suis arrivé à un résultat négatif..."

"Preuve de la divergence de la série"

"Divergence des séries de M. Linstedt"

"La divergence de ces développements n'aurait d'inconvénient que si l'on voulait s'en servir pour établir rigoureusement certains résultats, par exemple la stabilité du système solaire"

"...je cherche à expliquer en quoi consiste ce malentendu entre les géomètres et les astronomes; comment certaines séries que les premiers appellent divergentes peuvent rendre des services à ces derniers ..." 


\section{Annexe B. Le CAlCul COMme ÉCRITURe}

Nous baignons dans une époque à la fois de calcul et d'écriture. Et le "tout peut être écrit" semble se confondre au "tout peut être calculé". La puissance digitale a changé le monde [7].... tout comme les calculs des astronomes avaient changé le ciel au siècle précédant le dernier. Le credo : "On peut calculer tous les termes d'une série, donc la série existe (converge)" semble bien avoir été remplacé par "On peut tout mettre sur ordinateur, donc le monde est digital". Il s'agit bien d'écriture, puisque le codage par les nombres est une écriture.

Et une écriture qu'il faudra bien un jour déconstruire.

\section{Annexe C. Écriture et Changement de Paradigme en musique?}

La musique semble, tout comme la science. procéder par grands changements de paradigmes (apparition de la polyphonie, du développement, abandon de celui-ci, de la tonalité, etc). Il semble pourtant que le musicien fonctionne moins par souci de l'obstacle à surmonter, comme le fait le scientifique, que par souci du nouveau, du possible. Schönberg "démontre" que l'on peut écrire de la musique hors le cadre tonal, plutôt qu'il ne semble dépasser une certaine impasse de la modulation excessive (on pourrait cependant imaginer que la modulation infinie a un caractère "asymptotique" à l'atonalité, tout comme les séries de Poincaré le sont à la théorie du chaos). Pourtant le "Fuyons il va développer" de Debussy écoutant Brahms nous montre un musicien excédé, confronté à ce qui est pour lui un "résultat négatif" : la forme sonate (ou plutôt ce qu'elle est devenue). La méthodologie de Poincaré décrite dans cet article n'est certes pas la seule possibilité du progrès en sciences et bien des succès des mathématiques évitent cet affrontement direct (que Poincaré adorait, je pense) au négatif.

Pourtant si l'on est tenté de rapprocher Poincaré de Debussy, et beaucoup d'autres scientifiques de Schönberg, il me semble que l'abandon du développement en musique est resté intact, alors que l'atonalité a été bien vite remplacée par la série, elle même détonné quelques décennies plus tard.

Mais a une époque où les faiseurs de convergences (faiseurs d'anges?) tonale envahissent les institutions, peut-être serait-il bon de déconstruire un peu la tonalité, et de répondre à ceux qui posent encore et toujours la question : "pourquoi la musique de Schönberg est-elle si difficile à entendre?" par la question : "pourquoi est-il devenu impossible de composer de la musique tonale?".

\section{RÉFÉRENCES}

[1] G. Bachelard, "Essai sur la connaissance approchée", Vrin, Paris 1969.

[2] M.Born, "Vorlesungen über Atommechanik", Springer, Berlin, 1925. English translation : "The mechanics of the atom", Ungar, New-York, 1927.

[3] Fowler, "Statistical mechanics", Cambridge, 134-137, 1929.

[4] V. Jankélévitch, "Philosophie première. Introduction à une philosophie du presque", Gallimard, Paris, 1954. 
[5] N. Kolmogorov, "The general theory of dynamical systems and classical mechanics", Proceedings of the International Comgres of Mathematicians", Amsterdam 1954, North-Holland, 1957.

[6] G. Longo, Savoir critique et savoir positif : l'importance des résultats négatifs. Intellectica, 40, n.1, 2005 (english translation : "Information Technology and Media", web-page of the European Commission.)

[7] G. Longo et T. Paul, "Le monde et le calcul : rflexions sur calculabilit, mathmatiques et physique", "Logique \& Interaction : Gomtrie de la cognition" Actes du colloque et cole thmatique du CNRS "Logique, Sciences, Philosophie" Cerisy, Hermann, 2009.

[8] T. Paul, "Discrete-continuous and classical-quantum", "Mathematical Structures in Computer Science" 17, p. 177-183, (2007).

[9] T. Paul, "On the status of perturbation theory", "Mathematical Structures in Computer Science" 17, p. 277-288, (2007).

[10] T. Paul, "Poincaré et les quanta, Noésis (2011).

[11] H. Poincaré, "Les méthodes nouvelles de la mécanique céleste", Volume 3, Blanchard, Paris, 1987.

[12] H. Poincaré CEuvres, T 9., Gauthier-Villars, Paris (1954).

[13] H. Poincaré "Sur la théorie des quanta", CRAS Vol. 153, p. 1103-1008 (1911).

[14] H. Poincaré "Sur la théorie des quanta", J. de Physique théorique et appliquée, 5ième série, Vol. 2, p. 5-34 (1912).

[15] H. Poincaré "Sur le problèmes des trois corps et les équations de la dynamique", Act Mathematica, vol. 13, 5-165, 1890.

[16] J.M Yoccoz, "Une erreur féconde du mathématicien Henri Poincaré, Conférence à la BNF, (13 avril 2005), SMF, Gazette dse mathématiciens n 107, (janvier 2006).

CNRS et CMLS École polytechnique, 91128 Palaiseau Cedex

E-mail address: paul@math.polytechnique.fr 